\title{
Exact Weight Perfect Matching of Bipartite Graph Problem Simplified
}

\author{
THINH D. NGUYEN* \\ kosmofarmer@gmail.com
}

June 15, 2018

\begin{abstract}
The study of perebor dates back to the Soviet-era mathematics, especially in the 1980s [1]. Post-Soviet mathematicians has been working on many problems in combinatorial optimization. One of them is Exact Weight Perfect Matching of Bipartite Graph (EWPM). This particular problem has been thoroughly considered by [2], [3], [4]. In this note, we give a simpler proof about the solvability of EWPM.
\end{abstract}

\section{BACKGROUND, NOTATION AND PROPOSITIONS}

Natural number can be encoded in bi-
nary. Hamming weight of a binary
string is the number of 1's that it contains.

Lemma A game in that you can construct a multi-set of $m$ elements from $\left\{1,2, \ldots, 2^{m-1}\right\}$, duplicate(s) is (are) allowed. You win if the sum of your multi-set equals $2^{m}-1$. The only way to win is to construct a set which is the given set.

For reference on computer arithmetics, an excellent monograph is [5].

The-dimensional matching (3DM) is a combinatorial optimization. In [6], authors proves its intractability in details.

Lemma 3SAT $\leq_{p}$ 3DM

A keen reader should be able to realize that 3DM instances produced therein always have two sets of equal cardinality. One can note that the three sets are indexed by variable numbering and clause numbering or Cartesian product of these. Moreover, there are no two 3-tuple with more than one vertex in common.

\footnotetext{
* Perebor
}

For clarity, we give here the definition of EWPM as defined in [2].

Input An edge-weighted bipartite graph $G=(X \cup Y, E)$ with weight function $W: E \rightarrow$ $N$ and a natural number $\alpha$

Output 1 iff. there exists a perfect matching $\mathrm{M}$ with $W(M)=\alpha, 0$ otherwise

Proposition Under mathematics conjecture, EWPM cannot be solved efficiently.

If given a 3DM with two of three sets of equal cardinality, denote them by $X$ and $Y$, one can construct a EWPM instance $G=(X \cup Y, E)$ where $E$ is compatible with the $3 \mathrm{DM}$ instance. The weight function $W$ now reflects the "hidden" set. An edge connecting two vertices $u, v$ determines exactly one 3-tuple from the original instance. If the index of the "hidden" vertex is $i$ (zero-based), we weigh this edge $2^{i}$. Denote by $Z$ the "hidden set". The natural number $\alpha$ should be $2^{|Z|}-1$. At present, everything should work.

\section{CONCLUSION AND FUTURE VISION}

We can anticipate much work to be done on perebor in near future. Whether these colossal monuments can thrive in a (possibly) finite universe is of philosophical concerns. 


\section{REFERENCES}

[1] Phan Dinh Dieu, Le Cong Thanh, Le Tuan Hoa, Average Polynomial Time Complexity of Some NP-Complete Problems. Theor. Comput. Sci. 46(3): 219-237 (1986)

[2] Guohun Zhu, Xiangyu Luo, and Yuqing Miao, Exact Weight Perfect Matching of Bipartite Graph is NP-Complete, 2008

[3] Cameron, K., Coloured matchings in bipartite graphs, Discrete Math., V169, N1, pp. 205-209. 5/97

[4] Monnot, J., The labeled perfect matching in bipartite graphs, Information Processing Letters, V96, N3, pp. 81-88, 11/05

[5] Richard P. Brent, Paul Zimmerman, Modern Computer Arithmetic, Cambridge Monographs

[6] Michael R. Garey, David S. Johnson, COMPUTERS AND INTRACTABILITY. A Guide to the Theory of NP-Completeness, BELL LABORATORIES 